\title{
Symmetry breaking in binary Bose-Einstein condensates in the presence of an inhomogeneous artificial gauge field
}

\author{
S. Sahar S. Hejazi $\odot,{ }^{1, *}$ Juan Polo $\odot,{ }^{1}$ Rashi Sachdeva, ${ }^{2}$ and Thomas Busch $\oplus^{1}$ \\ ${ }^{1}$ Quantum Systems Unit, Okinawa Institute of Science and Technology Graduate University, Okinawa 904-0495, Japan \\ ${ }^{2}$ Mathematical Physics and NanoLund, LTH, Lund University, Box 118, 22100 Lund, Sweden
}

(Received 10 August 2020; accepted 20 October 2020; published 9 November 2020)

\begin{abstract}
We study a twocomponent Bose-Einstein condensate in the presence of an inhomogeneous artificial gauge field. In response to this field, the condensate forms a localized vortex lattice structure that leads to a nontrivial symmetry breaking in the phase separated regime. The underlying physical mechanism can be understood by considering the energy landscape and we present a simplified model that is capable of reproducing the main features of the phase separation transition. The intuition gained by numerically solving this simplified model is then corroborated using an analytical solution found within the Thomas-Fermi limit.
\end{abstract}

DOI: 10.1103/PhysRevA.102.053309

\section{INTRODUCTION}

Ultracold gases of neutral atoms have, in the past two decades, evolved into highly controllable systems that allow one to study and simulate numerous fundamental quantum mechanical effects [1-3]. One of the reasons for this is the large experimental toolbox for tuning almost all of the terms of their Hamiltonians using static or time-dependent external fields. This includes using spin-orbit coupling [4] or artificial gauge fields [5,6] to affect the kinetic part, optical lattices [2], density-dependent gauge potential [7] or painted potentials [8] to adjust the external trapping terms, or Feshbach resonances to control the nonlinear interaction terms [9], to name just a few. Additionally, systems with different symmetries can be created using multicomponent setups [10,11].

The simplest multicomponent system is a binary BoseEinstein condensate (BEC) made either from bosonic atoms in two different hyperfine states [12], two different isotopes [13], or two different elements [14-17]. These systems show intriguing physics related to interpenetrating superfluidity $[18,19]$ and in particular possess a demixing phase transition $[13,20-22]$ : in free space systems, the two components are miscible if $g_{12}^{2}<g_{11} g_{22}$, where the $g_{l l}$ are the intracomponent interaction strengths and $g_{12}$ is the inter-component one. Once this inequality condition is violated, the system phase separates. It is worth noting that for certain atomic condensate settings these three interaction strengths can in principle be tuned independently $[13,15]$. In nonhomogeneous systems, the point of the separation transition can be shifted, as the effects of the kinetic energy have to be taken into account [12,23-26]. Other terms that can be present in the Hamiltonian, e.g., accounting for Rabi coupling [27], spinorbit coupling [28], or rotations [7,29], are known to have an influence on the phase separation threshold as well.

\footnotetext{
*sahar.hejazi@oist.jp
}

In this work, we are interested in the phase separation process in a two-component system in the presence of angular momentum. However, contrary to previously considered situations $[19,30]$, we will investigate systems where the rotational energy is not homogeneously distributed over the whole condensate. While such a situation can in principle be realized experimentally by locally creating vortices through phase imprinting [31,32], this technique usually leads to nonequilibrium situations as the condensate has to adjust its density to accommodate the imprinted phase distribution. To avoid excitations, such as phonon modes, which can have non-negligible effects on the phase-separation transition [33], we instead consider spatially inhomogeneous artificial gauge fields that only induce rotation in certain areas of the condensate [34-38].

Condensates in harmonic traps have been shown to respond to homogeneous rotation with the formation of triangular vortex lattices [39], whereas in different external potentials different geometrical arrangements of the vortices are possible [40-43]. Furthermore, condensates that encompass low-density regions, either due to local potential maxima or in the phase separated limit of a multicomponent system, can support the so called hidden or ghost vortices located in these regions $[29,30,44-47]$. While the response to localized rotation through a gauge field has already been explored for single-component condensates [35-37], the effect on the phase separation transition in two-component systems has not yet been discussed.

In order to clearly isolate the effects of localized rotation, we reduce the system's degrees of freedom by considering that both condensates are made from atoms of the same species, both have the same number of particles and both have identical intracomponent interaction strengths. We also restrict ourselves to a fundamental two-dimensional dynamics and a rotationally isotropic trapping geometry. Without rotation the separation transition in such a system leads to a straight phase boundary that cuts through the center of the trapping potential and whose direction is due to spontaneous symmetry breaking. In the presence of strong, global external rotation, 
this is no longer the case and the phase separation dynamics becomes highly complex and breaks all spatial symmetries by forming unordered serpentine vortex sheets [29].

To create a situation which lies in between the nonrotating and globally rotating settings we consider a gauge field that originates from an evanescent optical field above the surface of a prism, close to which a two-component condensate is trapped. The short-range exponential decay of the evanescent field in the direction perpendicular to the prism surface then results in an artificial magnetic field with a pronounced maximum at some distance from the surface. While in the miscible regime this produces a localized vortex distribution in the direction parallel to the surface that is, in principle, consistent with the symmetric splitting of the two components, we show that the interplay between the kinetic and the interaction energy leads to additional symmetry breaking that is not purely determined by minimising the length of the phase boundary.

This manuscript is organized as follows. In Sec. II, we describe our model for a two-component Bose-Einstein condensate in the presence of a nonhomogeneous artificial gauge field originating from the evanescent field created at the surface of a dielectric prism. In Sec. III, we show how this artificial gauge field affects the miscible and immiscible regimes and in Sec. IV, we study, through a simplified model, the physical mechanisms behind the symmetry breaking observed in the immiscible regime. The numerical results obtained are then corroborated using an analytical Thomas-Fermi model in Sec. V, and finally we conclude in Sec. VI.

\section{MODEL}

We consider a two-component Bose-Einstein condensate of neutral alkali atoms that is tightly confined in one spatial direction, such that it can be effectively treated using a two-dimensional description [48]. In particular, we choose a harmonic trapping potential of the form $V(x, z)=$ $\frac{1}{2} M \omega^{2}\left(x^{2}+z^{2}\right)$, where the frequency $\omega$ is the same in both directions, so that the trap is symmetric in the $x-z$ plane. Furthermore we assume that the atoms in both components have the same mass $M$, which can be achieved by trapping a single species and condensing the atoms in two different internal states. For simplicity we assume equal and conserved numbers of particles in each component. The entire system is located just above the surface of a dielectric prism with refractive index $n$, so that the atoms can interact with the evanescent field, see Fig. 1.

Within the mean field approach, the two-component BEC can be described by a set of coupled Gross-Pitaevskii equations (GPEs) of the form [49]

$i \hbar \frac{\partial \phi_{l}}{\partial t}=\left[\frac{1}{2 M}\left(P_{l}-\mathbf{A}\right)^{2}+V(x, z)+g_{l l}\left|\phi_{l}\right|^{2}+g_{l m}\left|\phi_{m}\right|^{2}\right] \phi_{l}$,

where $g_{l l}=\frac{4 N \pi \hbar^{2} a_{l}}{M}$ and $g_{l m}=\frac{4 N \pi \hbar^{2} a_{l m}}{M}$ are the intra- and intercomponent scattering strengths, respectively, with $l, m=$ $\{1,2\}$ and $l \neq m$. As usual, $a_{l}$ is the $s$-wave scattering length between atoms of the same component and $a_{l m}$ for atoms of different components. The condensate wave function is described by $\phi_{l}$ and is normalized as $\iint\left|\phi_{l}\right|^{2} d x d z=1$.

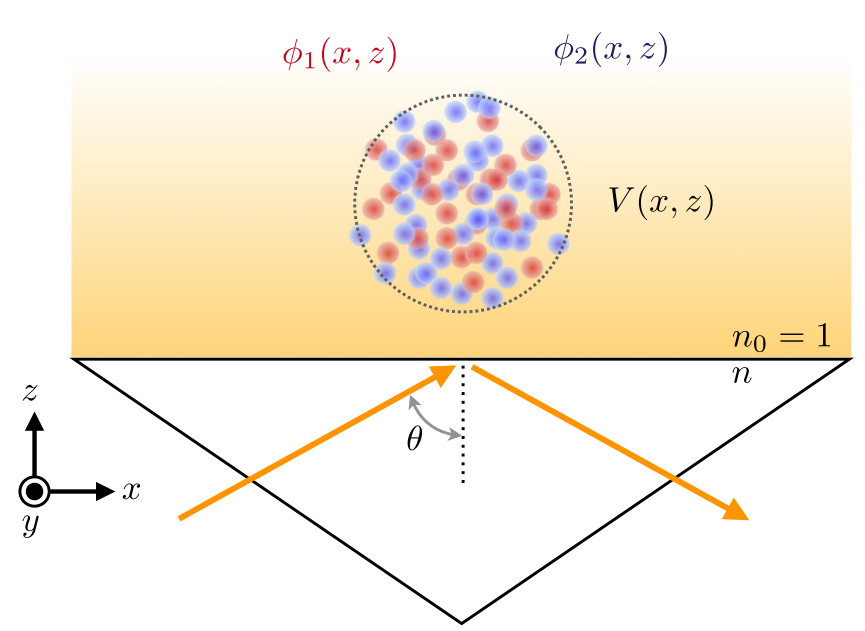

FIG. 1. Schematic representation of a two-component BEC trapped in a potential $V(x, z)$ (geometry indicated by the dashed circle) just above the surface of a dielectric prism with the refractive index $n$. The center of both BECs is located at the origin of the coordinate system and and we always assume the surface of the prism to be at $z / a_{0}=-10$.

The vector field $\mathbf{A}$ represents the gauge potential, which stems from the evanescent field emanating from the prism surface.

To describe the gauge field we consider a laser field with a wave vector $\mathbf{k}$ and frequency $\omega_{L}$, chosen to be close to the resonance of the atomic transition. This field propagates inside the prism at an angle $\theta$ with respect to its surface. When this angle is larger than the critical angle, $\theta_{0}=\arcsin \left(\frac{1}{n}\right)$, the beam undergoes total internal reflection and an evanescent field is created at the surface of the prism. The electric field, $\mathbf{E}(x, z, t)$, propagates in $x-z$ plane with an amplitude $\mathbf{E}_{0}$ and decays from the surface in the positive $z$ direction with a penetration depth $d=\left(k_{0} \sqrt{n^{2} \sin ^{2} \theta-1}\right)^{-1}$. It takes the form

$$
\mathbf{E}(x, z, t)=t^{\mathrm{TE}}(\theta) \mathbf{E}_{0} e^{-i\left(\omega_{L} t-\varphi(x)\right)} e^{-z / d},
$$

where $t^{\mathrm{TE}}(\theta)=2 n \cos \theta\left(n \cos \theta+i \sqrt{n^{2} \sin ^{2} \theta-1}\right)^{-1}$ corresponds to the transmission coefficient, and the running phase is given by $\varphi(x)=x k_{0} n \sin \theta$ [36].

The interaction between the evanescent field and the atoms in the condensate occurs via dipole coupling, $\mathbf{d} \cdot \mathbf{E}(x, z)$, where $\mathbf{d}$ is the electric dipole moment of the atoms. Without loss of generality we assume it to be the same for both components. In the rotating wave approximation this then leads to a dressed state of the form [36]

$$
|\chi(x, z)\rangle=\left(\begin{array}{l}
\cos [\Phi(x, z) / 2] \\
\sin [\Phi(x, z) / 2] e^{-i \varphi(x)}
\end{array}\right),
$$

where $\Phi(x, z)=\arctan \left(\frac{|\kappa(x, z)|}{\Delta}\right), \kappa(x, z)=\mathbf{d} \cdot \mathbf{E}(x, z) / \hbar$ and $\Delta=\omega_{L}-\omega_{A}$ is the detuning of the laser light from the atomic resonance frequency, $\omega_{A}$, which we assume again to be the same for both components. Assuming that the atoms move slowly enough to adiabatically follow this spatially inhomogeneous eigenstate, they pick up a geometrical Berry phase which can be written as the appearance of a vector potential 
(a)

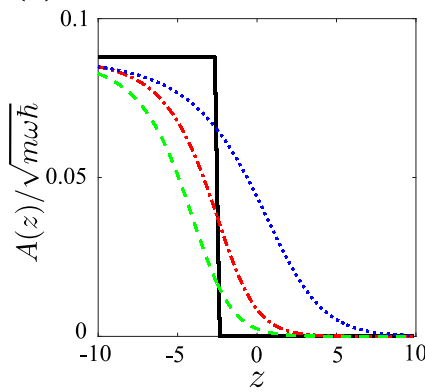

(b)

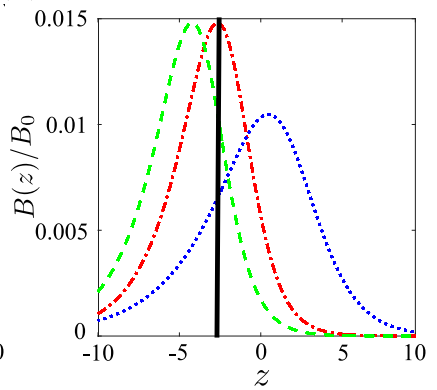

FIG. 2. (a) Gauge potentials as a function of the distance above the prism surface. The green (dashed) and red (dotdash) line correspond to an incident angle of $\theta-\theta_{0}=8 \times 10^{-4} \mathrm{rad}$, and $s=10$ and $s=20$, respectively. The blue (dotted) curve corresponds to $\theta-\theta_{0}=4 \times 10^{-4} \mathrm{rad}$ for $s=20$. The black step function represents the model used in Sec. IV. (b) Normalized magnetic fields, $B(z) / B_{0}$, corresponding to the gauge potentials shown in (a) with the same color coding, as plotted in [36]. Note that $z$ is given in harmonic oscillator units.

$\mathbf{A}=i \hbar\langle\chi \mid \nabla \chi\rangle$, which has the explicit form

$$
\begin{aligned}
\mathbf{A}(x, z) & =\hbar \sin ^{2}[\Phi(z) / 2] \nabla \phi(x) \\
& =\frac{n \hbar k_{0}}{2}\left[1-\frac{1}{\sqrt{1+\left|\frac{\kappa(x, z)}{\Delta}\right|^{2}}}\right] \sin \theta \hat{\mathbf{x}} .
\end{aligned}
$$

The condition for this adiabatic following is that the atomic velocity is limited by $v \ll \Omega /\left(\left|\left\langle\Psi_{2} \mid \nabla \Psi_{1}\right\rangle\right|\right)$, where $\Omega=$ $\sqrt{\Delta^{2}+|\kappa|^{2}}$ is a generalized Rabi frequency [50]. An artificial magnetic field can then be calculated from the vector potential via $\mathbf{B}=\nabla \times \mathbf{A}$ as [36]

$$
\mathbf{B}(x, z)=-B_{0} \sqrt{n^{2} \sin ^{2} \theta-1} \frac{s^{2} \beta(z) n \sin \theta}{\left[1+s^{2} \beta(z)\right]^{3 / 2}} \hat{\mathbf{y}},
$$

with $B_{0}=\hbar k_{0}^{2} / 2, \quad \beta(z)=\left|t^{(\mathrm{TE})}(\theta)\right|^{2} e^{-2 z / d}$ and $s=\frac{\left|\mathbf{d} \cdot \mathbf{E}_{0}\right|}{\hbar|\Delta|}$. From Eqs. (4) and (5) one can directly see that, since the evanescent field decays with increasing distance above the surface of the prism, the gauge field and the $B$ field will have to be inhomogeneous as well. However, note that both fields have a trivial dependence in the $x$ direction. For given sets of laser parameters this is shown in Fig. 2(a) for the $A$ field and in Fig. 2(b) for the $B$ field. In particular, one can see from these plots that the artificial magnetic field has a maximum at finite distance away from the surface. The position of this maximum strongly depends on the value of $s$, while a change in the angle of the incident beam mostly affects the amplitude of the magnetic field. The atoms in a condensate trapped within the evanescent field will therefore experience effects corresponding to the presence of a spatially inhomogeneous $B$ field $[35,36]$.

\section{EFFECTS OF THE INHOMOGENEOUS MAGNETIC FIELD}

To explore the effects of the inhomogeneous artificial gauge potential, we numerically solve the two coupled

(a)

(b)
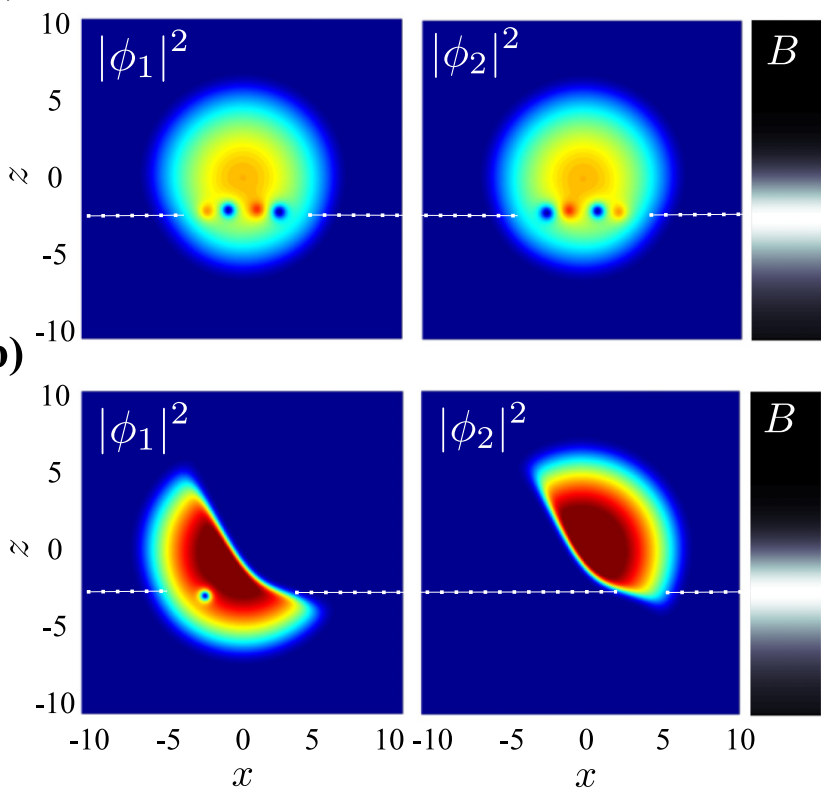

FIG. 3. Ground state density profiles for the condensate trapped in the evanescent field in (a) the miscible ( $\alpha=0.5)$ and (b) immiscible $(\alpha=1.5)$ regimes. We choose the parameter $s=20$ and a laser field that has an incident angle of $\theta-\theta_{0}=8 \times 10^{-4}$ rad with respect to the prism surface. The intensity of the $B$ field in the $x$ and $z$ direction is indicated on the right-hand side. The artificial magnetic field is generated by choosing $k_{0} a_{0}=1.0871 \times 10^{7}$ and $\kappa(x, z) / \Delta=20$. Spatial coordinates $x$ and $z$ are expressed in harmonic oscillator units.

Gross-Pitaevskii equations (1) by using a standard FFT/splitoperator method [51]. From here onward, we work in harmonic oscillator units, that is $x \rightarrow x / a_{0}, z \rightarrow z / a_{0}$ and $t \rightarrow t \omega$, with $a_{0}=\sqrt{\hbar / M \omega}$. We choose equal intracomponent coupling coefficient, $g_{11}=g_{22}=g$ [12] and intercomponent coupling is given by $g_{12}=g_{21} \equiv \alpha g$. For stability reasons, we only consider repulsive interactions. Thus the condensate is in the miscible regime for $0<\alpha \lesssim 1$, and in the phase separated regime for $\alpha \gtrsim 1$.

In Fig. 3, we show examples of ground state density profiles within the miscible and immiscible regimes. One can immediately note that the vortices only appear in a localized area, which corresponds to the region where the $B$ field is largest $[35,36]$. In the miscible regime and for the parameters chosen in Fig. 3, they form a single line along the maximum of the $B$ field, with each component having an offset with respect to the other such that they minimize the interaction energy. However, for less localized $B$ fields they can also arrange in a localized triangular lattices that converges to the full Abrikosov geometry for global fields [36].

While in the immiscible regime the condensate components separate, as expected, it is immediately clear from Fig. 3(b) that the separation can not be driven by the minimization of the interaction energy alone. Naively one could expect that the separation boundary would be a straight line along the $z$ direction at $x=0$, which would lead to minimising the interaction energy and the kinetic energy stemming from the boundary, while ensuring that both condensates have the same amount of vortices and energy. However, this would not 
necessarily minimize the overall energy of the system, as additional kinetic energy is associated with the vortices. In fact, Fig. 3(b) shows that only one of the components carries visible vortices and that, even though the external parameters are the same for both situations, the number of vortices is not the same in the miscible and the immiscible regimes. This clearly indicates that some of the vorticity in the system disappeared in favor of the so-called ghost vortices, which are located in the low density areas, so that the large rotational energy required to rotate high densities is avoided. To understand the interplay between the interaction and rotational energy in more detail, we will in the following explore a toy model of the inhomogeneous $B$ field that captures all relevant processes.

\section{TOY MODEL}

Since the main characteristic of the inhomogeneous magnetic field is the existence of a localized maximum [see Fig. 2(b)], we will in the following consider the limit where the $B$ field is tightly localized in space. This can be achieved by assuming a steplike gauge-potential given by

$$
\mathbf{A}_{\Theta}(z)=A_{0} \Theta\left(-z+z_{0}\right),
$$

where $\Theta(z)$ is the Heaviside step function, $z_{0}$ is the shift of the Heaviside function in the $z$ direction, and $A_{0}$ is the strength of the artificial gauge potential [see Fig. 1(b)]. The field is constant in the $x$ direction. This form of the gauge potential captures the physical parameters that are related to the evanescent electric field created at the surface of the prism in a physically realistic and clean way: $A_{0}$ accounts for all the experimental parameters that characterize the strength of the realistic gauge potential [see Eq. (4)] and $z_{0}$ accounts for the shift in real space due to the $s$ parameter. In all our simulations below we chose $A_{0}$ such that its maximum value has the same order of magnitude as the realistic model.

Typical ground state density distributions in the miscible and phase-separated regimes of the two-component system are shown in Fig. 4. In the miscible regime, $\alpha<1$, the localized $B$ field leads to a single-line of vortices, see Fig. 4(a). Due to the repulsive interactions between components, vortices within each component arrange themselves with an offset with respect to their counterparts, effectively filling the low density vortex cores of the other component. This is very similar to the realistic setting considered above, see Fig. 3(a).

For the phase separation regime, $\alpha \gtrsim 1$, we show in Figs. 4(b)-4(d) the density distribution for three different values of $z_{0}$. In panel (c), the $B$ field is located exactly at the center of the $\operatorname{BEC}\left(z_{0}=0\right)$, and one can see that this leads to a separation of the two components into two clouds with essentially mirror-symmetric density profiles. The phase boundary is exactly along the line of the finite $B$ field and corresponds to the shortest line possible. No vortices are visible and all vorticity is removed from the system, leading to ghost-vortices located in the low density areas $[29,30]$. This solution clearly minimizes the interaction and the kinetic energy of the system and is reminiscent of the standard phase separation in twocomponent systems without vorticity. However, the direction of symmetry breaking is now determined by the $B$ field and not chosen spontaneously. (a)
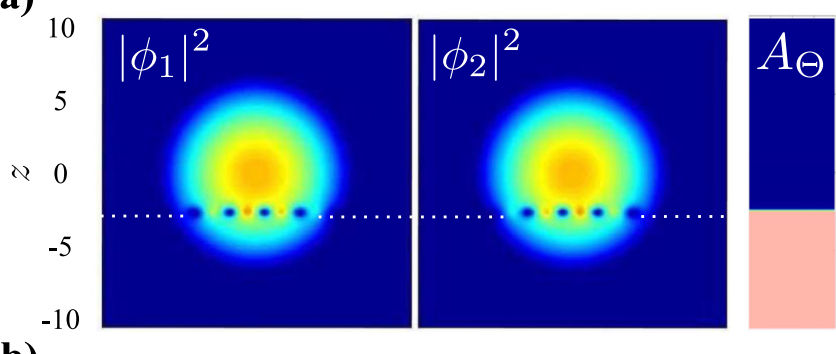

(b)

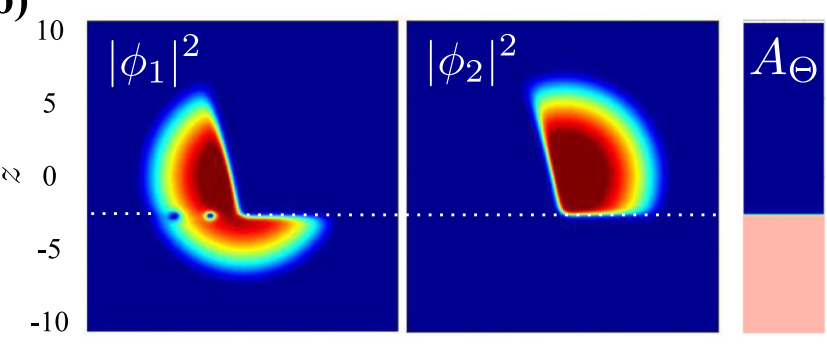

(c)

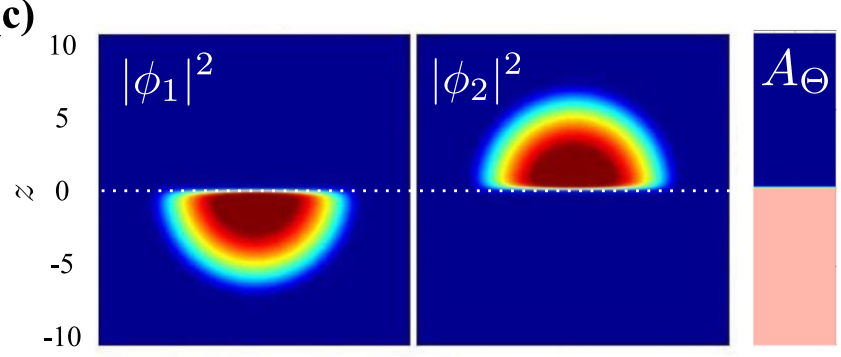

(d)

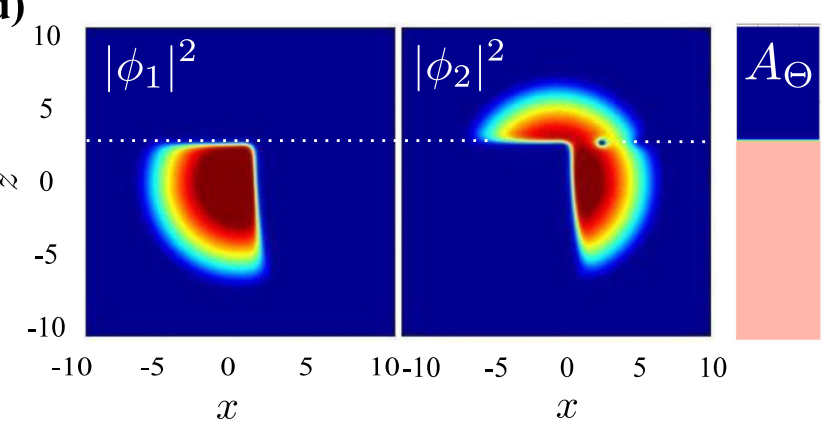

FIG. 4. Ground state density profiles of each component of the two-component BEC in the presence of the step-function gauge potential (indicated on right-hand side: light peach color corresponds to finite value of $A_{0}=0.3$ and dark blue to zero). (a) Miscible regime with $\alpha=0.5$ and $z_{0}=-2.5$. [(b)-(d)] Immiscible regime with $\alpha=$ 1.5 and $z_{0}=-2.5,0$, and 2.5 , respectively. All density plots use the same color scale. Spatial coordinates $x$ and $z$ are expressed in harmonic oscillator units.

For $z_{0} \neq 0$, this simple picture breaks down and the additional kinetic energy in the system plays a crucial role in how the phase separation occurs. In Figs. 4(b) and 4(d), we show the density profiles for $\alpha=1.5$ and the $B$ field located at $z_{0}=-2.5$ and 2.5 , respectively. One can immediately see that the two components separate in a nonsymmetric way, which strongly depends on the position of the $B$ field, and that one component still possesses vortices, while the other does not. In particular one can see that the phase boundary is only partly along the line of the $B$ field, before turning to be more aligned along the $x$ direction. The part along the $z$ direction 
(a)

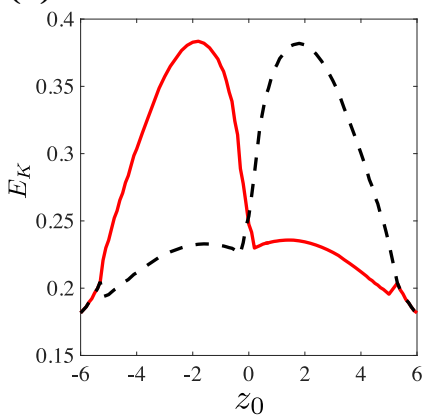

(b)

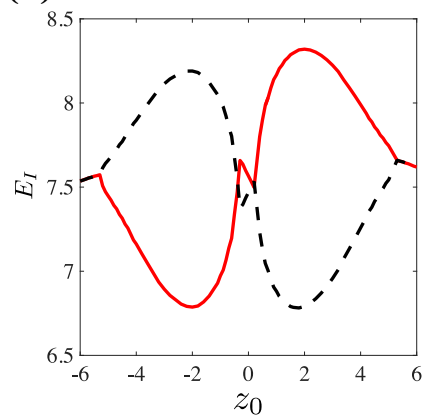

FIG. 5. (a) Kinetic energy $E_{\mathrm{K}}^{(l)}$ and (b) interaction energy $E_{\mathrm{I}}^{(l)}$ as a function of the dimensionless position of the artificial magnetic field $z_{0}$, for both components of the BEC in the immiscible regime $(\alpha=$ 1.5). The red (solid) line corresponds to the energy of first component and black (dashed) line to the energy of the second component. The artificial magnetic field is fixed at $A_{0}=0.3$ in harmonic oscillator units.

increases in length with increasing $z_{0}$, becomes the length of the whole condensate at $z_{0}=0$, and then decreases again almost symmetrically for $z_{0}>0$. While this symmetry breaking behavior seems unusual at first sight, it can be intuitively understood by realising that the system is still trying to reduce the rotational energy by creating ghost vortices in low density area. Yet, when the $B$-field line does not cross the condensate symmetrically, separating the components fully along this line would lead to one component having a significantly smaller area available compared to the other. As the interaction energy is nonlinear, this would lead to a significant increase in the overall energy, which is unfavorable. Thus the system uses the $B$-field line partly to minimize the rotational energies, but then minimzes the interaction energies by departing from it. The phase separation is therefore a careful balance between the minimization of the interaction and the rotational energies. It is important to realize that the situations for values of $z_{0}$ that are symmetric around zero are not fully identical [see Figs. 4(b) and 4(c)], as the $A$ field breaks the system's symmetry along $z$. Again, this is qualitatively the same behavior that is also found in the realistic model shown in Fig. 3(b).

The intuition for the immiscible regime developed above can be supported by looking at the kinetic and interaction energy of each component given by

$$
\begin{aligned}
& E_{\mathrm{K}}^{(l)}=\frac{1}{2} \iint \phi_{l}^{*}\left(i \nabla_{l}+\mathbf{A}_{\Theta}\right)^{2} \phi_{l} d x d z, \\
& E_{\mathrm{I}}^{(l)}=\frac{g}{2} \iint\left(\left|\phi_{l}\right|^{4}+\alpha\left|\phi_{m}\right|^{2}\left|\phi_{l}\right|^{2}\right) d x d z .
\end{aligned}
$$

One can see from Fig. 5 that the kinetic energy of the vortexcarrying component grows initially much faster as the $B$ field line moves through the condensate, compared to that of the second component. However, with increasing values of $z_{0}$, the length of the phase separation border along the $B$ field line grows, leading to more and more vortices turning into ghost vortices. The kinetic energy therefore decreases again, until the same value is reached for both components when $z_{0}=0$, i.e., when all vortices have become ghost vortices. The same process then repeats in the second component, which starts carrying the vortices once $z_{0}>0$.

The graph of the interaction energy as a function of $z_{0}$ [see Fig. 5(b)] shows that the component carrying the vortices has generally a lower nonlinear energy than the one that carries no angular momentum. This is due to the additional centrifugal forces in the vortex carrying component, which allow the system to achieve a lower density. Again, these variations in energy go to zero when all vortices have been turned into ghost vortices at $z_{0}=0$ and the role of the two components flips subsequently. It is worth noting that when the $B$ field passes $z_{0}=0$ a jump in the interaction energy can be seen as there exist a sudden point when the last visible vortex has been turned into a ghost vortex. Again, the asymmetries present in the kinetic and interaction energies are due to the effect of the $A$ field, which increases the total energy of the system as it increasingly envelopes the entirety of the twocomponent BEC.

\section{SYMMETRY BREAKING}

While the part of the phase separation line along the $B$ field line is set by external parameters, the remaining question is about the position of the turning point and the direction of the break away from it. Intuitively it should be as short as possible, which for rotationally isotropic geometries should lead to a break at a right angle.

One can see from Figs. 4(b)-4(d) that this is approximately the case and below we confirm this intuition by determining the break-off point $\left(x_{0}, z_{0}\right)$ by energy minimization using the Thomas-Fermi (TF) wave function, $\phi_{l}^{\mathrm{TF}}$, obtained from solving

$$
\mu_{l}^{\mathrm{TF}} \phi_{l}^{\mathrm{TF}}=\left[V(x, z)+g\left|\phi_{l}^{\mathrm{TF}}\right|^{2}+\alpha g\left|\phi_{m}^{\mathrm{TF}}\right|^{2}\right] \phi_{l}^{\mathrm{TF}},
$$

where $\mu_{l}^{\mathrm{TF}}$ is the chemical potential of each component and we are again using harmonic oscillator units $[23,24,52]$. This approximation is valid when the kinetic energy terms of the Hamiltonian can be neglected as they are much smaller than the nonlinear ones (see Fig. 4).

However, as the kinetic energy clearly plays an important role in the phase separation, we take its effect into account by fixing the phase separation line along the maximum of the magnetic field in the $x$ direction up to a value of $x_{0}$. We then approximate the rest of the phase separation border by a straight line along the $z$ direction, so that both parts have a sharp $\pi / 2$ angle between them (see inset of Fig. 6). These conditions are encoded in the limits of integration for all integrals which depend on the position of the vertical part of the phase boundary, i.e., $x_{0}$. For simplicity, we also fix the TF radius to $R_{\mathrm{TF}}=\sqrt{2 \sqrt{g / \pi}}$ in all limits of integration, so that no extra functional dependence on $x_{0}$ or $z_{0}$ appears. This allows us to avoid coupled transcendental equations for the chemical potentials of both components. Therefore, in the case presented here, we find analytical expressions for the wave functions within the TF model, with an ansatz for the artificial gauge field encoded in the boundary conditions of the model.

Within this model, we then minimize the total energy of the two-component system as a function of the position of 
(a)

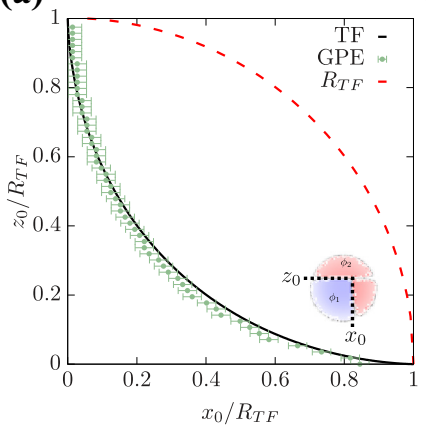

(b)

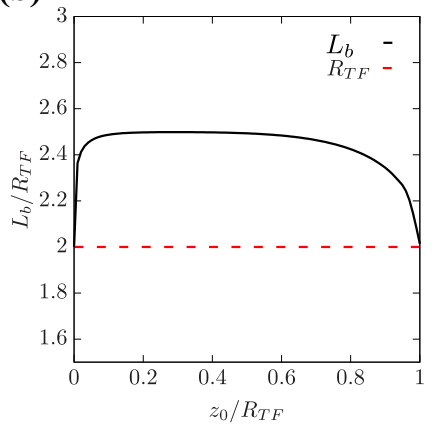

FIG. 6. (a) Turning points $\left(x_{0}, z_{0}\right)$ of the asymmetric phase separation (see Fig. 4 for density plots) comparing the analytical Thomas-Fermi solution given by the solid-black line with the GPE numerical simulations, green dots (see Ref. [53] for details) for $\alpha=1.5$. This plot shows the first quadrant of the $2 \mathrm{D}$ representation of the BEC, with $(0,0)$ corresponding to the center of the harmonic trap. The dashed-red line identifies the area delimited by the ThomasFermi radius found at $\alpha=0$. (b) Length of the phase boundary, $L_{b}$, within the TF approximation as a function of the position of the maxima of the magnetic field $z_{0}$. The dashed-red line indicates the shortest path for the phase boundary, i.e., $2 R_{T F}$.

the breaking point from the phase separation along the $B$ field line, at $x_{0}$. This is given by an analytical expression of the minimized energy as a function of $x_{0}$. The results from this analytical approach are shown in Fig. 6(a) and one can immediately see that they very closely match the ones found from numerically solving the full two-component GPEs (see Ref. [53] for details). This indicates that the straight line boundary along the $x$ direction connected at a right angle to the first part of the boundary along $z_{0}$ provides the lowest energy solution for the system to phase separate and forces it to spontaneously break the symmetry. We also show in Fig. 6(b) that the length of the phase boundary exceeds the diameter of the condensate whenever the position of the $B$ field breaks the symmetry of the system. It is worth noting that the position where the symmetry breaking happens makes a mirror-symmetric curve to the TF boundary. This behavior of the symmetry breaking can be traced back, within the simplified TF model, to the symmetry imposed by the harmonic trap, while exhibiting nonetheless an excellent agreement with the numerical results of the TCGPE.

Finally, it is worth noting that while the assumption of a right angle connection between the two parts of the phase boundary is a good assumption in the toy model of the gauge field, it only approximately holds in the realistic model discussed in Sec. III. This is due to the $B$ field being spread out over a larger range in the $z$-direction and the vortices being discrete along the $B$-field line.

\section{CONCLUSIONS}

In this work, we have investigated the ground state of a two-component Bose-Einstein condensate in the presence of an inhomogeneous artificial gauge potential. This situation appears when the gauge field is created by an evanescent field at the surface of a dielectric material, close to which the BEC is trapped, and it is therefore experimentally realistic. While in the miscible regime, the system responds to the angular momentum imposed by the gauge field in an expected manner. In the phase separated regime a careful balance between the need to minimize the interaction and the kinetic energy leads to a phase separation that spontaneously breaks the symmetry in unusual ways. In particular, the phase separation border is no longer just a straight line that crosses the system symmetrically as would be the case in the absence of the gauge field.

Using a toy model, we have carefully explored the mechanism behind this symmetry breaking and clearly described the importance of the kinetic energy in the phase separation process of the two-component system. To confirm our numerical results, we have also presented an energy minimization calculation using the analytical TF solution that allows to determine the position where the phase boundary turns away from being a straight line.

One interesting extension of the above work would be to consider BECs that do not have equal number of particles or equal intra-component interaction strengths. Such systems can lead to different phase separation scenarios, such as radial geometries in harmonic traps. Combining a radial symmetry with localized, no-radially symmetric gauge fields would allow one to explore an even larger set of possible ways to break the symmetry.

Using inhomogeneous gauge potentials to induce local rotation into condensates holds the potential to be a valuable way to engineer and study interesting superfluid dynamics. These can range from the above study on phase separation in multicomponent condensates to creating well-defined initial states to study quantum turbulence [54]. The fact that such systems are experimentally possible using today's technology makes this an exciting and promising direction of research.

\section{ACKNOWLEDGMENTS}

This project was supported by Okinawa Institute of Science and Technology Graduate University. J.P. also acknowledges the JSPS KAKENHI Grant No. 20K14417.
[1] M. Lewenstein, A. Sanpera, V. Ahufinger, B. Damski, A. Sen(De), and U. Sen, Ultracold atomic gases in optical lattices: Mimicking condensed matter physics and beyond, Adv. Phys. 56, 243 (2007).

[2] I. Bloch, J. Dalibard, and W. Zwerger, Many-body physics with ultracold gases, Rev. Mod. Phys. 80, 885 (2008).
[3] I. Bloch, J. Dalibard, and S. Nascimbene, Quantum simulations with ultracold quantum gases, Nat. Phys. 8, 267 (2012).

[4] Y. Zhang, M. E. Mossman, Th. Busch, P. Engels, and C. Zhang, Properties of spin-orbit-coupled Bose-Einstein condensates, Front. Phys. 11, 118103 (2016). 
[5] J. Dalibard, F. Gerbier, G. Juzeliūnas, and P. Öhberg, Colloquium: Artificial gauge potentials for neutral atoms, Rev. Mod. Phys. 83, 1523 (2011).

[6] N. Goldman, G. Juzeliūnas, P. Öhberg, and I. B. Spielman, Light-induced gauge fields for ultracold atoms, Rep. Prog. Phys. 77, 126401 (2014).

[7] M. Edmonds and M. Nitta, Vortex patterns of atomic boseeinstein condensates in a density-dependent gauge potential, Phys. Rev. A 102, 011303(R) (2020).

[8] K. Henderson, C. Ryu, C. MacCormick, and M. G. Boshier, Experimental demonstration of painting arbitrary and dynamic potentials for Bose-Einstein condensates, New J. Phys. 11, 043030 (2009).

[9] C. Chin, R. Grimm, P. Julienne, and E. Tiesinga, Feshbach resonances in ultracold gases, Rev. Mod. Phys. 82, 1225 (2010).

[10] S. Taie, Y. Takasu, S. Sugawa, R. Yamazaki, T. Tsujimoto, R. Murakami, and Y. Takahashi, Realization of a SU(2) $\times \mathrm{SU}(6)$ System of Fermions In a Cold Atomic Gas, Phys. Rev. Lett. 105, 190401 (2010).

[11] S. Capponi, P. Lecheminant, and K. Totsuka, Phases of onedimensional SU(N) cold atomic Fermi gases - From molecular Luttinger liquids to topological phases, Ann. Phys. 367, 50 (2016).

[12] C. J. Myatt, E. A. Burt, R. W. Ghrist, E. A. Cornell, and C. E. Wieman, Production of Two Overlapping Bose-Einstein Condensates by Sympathetic Cooling, Phys. Rev. Lett. 78, 586 (1997).

[13] S. B. Papp, J. M. Pino, and C. E. Wieman, Tunable Miscibility in a Dual-Species Bose-Einstein Condensate, Phys. Rev. Lett. 101, 040402 (2008).

[14] D. J. McCarron, H. W. Cho, D. L. Jenkin, M. P. Köppinger, and S. L. Cornish, Dual-species Bose-Einstein condensate of ${ }^{87} \mathrm{Rb}$ and ${ }^{133} \mathrm{Cs}$, Phys. Rev. A 84, 011603(R) (2011).

[15] G. Thalhammer, G. Barontini, L. De Sarlo, J. Catani, F. Minardi, and M. Inguscio, Double Species Bose-Einstein Condensate with Tunable Interspecies Interactions, Phys. Rev. Lett. 100, 210402 (2008).

[16] G. Ferrari, M. Inguscio, W. Jastrzebski, G. Modugno, G. Roati, and A. Simoni, Collisional Properties of Ultracold K-Rb Mixtures, Phys. Rev. Lett. 89, 053202 (2002).

[17] G. Modugno, M. Modugno, F. Riboli, G. Roati, and M. Inguscio, Two Atomic Species Superfluid, Phys. Rev. Lett. 89, 190404 (2002).

[18] E. Fava, T. Bienaimé, C. Mordini, G. Colzi, C. Qu, S. Stringari, G. Lamporesi, and G. Ferrari, Observation of Spin Superfluidity in a Bose Gas Mixture, Phys. Rev. Lett. 120, 170401 (2018).

[19] L. Mingarelli and R. Barnett, Exotic Vortex Lattices in Binary Repulsive Superfluids, Phys. Rev. Lett. 122, 045301 (2019).

[20] M. Cipriani and M. Nitta, Crossover Between Integer and Fractional Vortex Lattices in Coherently Coupled Two-Component Bose-Einstein Condensates, Phys. Rev. Lett. 111, 170401 (2013).

[21] E. Nicklas, H. Strobel, T. Zibold, C. Gross, B. A. Malomed, P. G. Kevrekidis, and M. K. Oberthaler, Rabi Flopping Induces Spatial Demixing Dynamics, Phys. Rev. Lett. 107, 193001 (2011).

[22] U. Shrestha, J. Javanainen, and J. Ruostekoski, Pulsating and Persistent Vector Solitons in a Bose-Einstein Condensate in a Lattice Upon Phase Separation Instability, Phys. Rev. Lett. 103, 190401 (2009).
[23] T.-L. Ho and V. B. Shenoy, Binary Mixtures of Bose Condensates of Alkali Atoms, Phys. Rev. Lett. 77, 3276 (1996).

[24] H. Pu and N. P. Bigelow, Properties of Two-Species Bose Condensates, Phys. Rev. Lett. 80, 1130 (1998).

[25] E. Timmermans, Phase Separation of Bose-Einstein Condensates, Phys. Rev. Lett. 81, 5718 (1998).

[26] L. Wen, W. M. Liu, Y. Cai, J. M. Zhang, and J. Hu, Controlling phase separation of a two-component Bose-Einstein condensate by confinement, Phys. Rev. A 85, 043602 (2012).

[27] I. M. Merhasin, B. A. Malomed, and R. Driben, Transition to miscibility in a binary Bose-Einstein condensate induced by linear coupling, J. Phys. B: At., Mol. Opt. Phys. 38, 877 (2005).

[28] S. Gautam and S. K. Adhikari, Phase separation in a spin-orbitcoupled Bose-Einstein condensate, Phys. Rev. A 90, 043619 (2014).

[29] K. Kasamatsu, M. Tsubota, and M. Ueda, Vortex Phase Diagram in Rotating Two-Component Bose-Einstein Condensates, Phys. Rev. Lett. 91, 150406 (2003).

[30] K. Kasamatsu and K. Sakashita, Stripes and honeycomb lattice of quantized vortices in rotating two-component Bose-Einstein condensates, Phys. Rev. A 97, 053622 (2018).

[31] L. Dobrek, M. Gajda, M. Lewenstein, K. Sengstock, G. Birkl, and W. Ertmer, Optical generation of vortices in trapped BoseEinstein condensates, Phys. Rev. A 60, R3381 (1999).

[32] L. J. O'Riordan and Th. Busch, Topological defect dynamics of vortex lattices in Bose-Einstein condensates, Phys. Rev. A 94, 053603 (2016).

[33] K. L. Lee, N. B. Jørgensen, L. J. Wacker, M. G. Skou, K. T. Skalmstang, J. J. Arlt, and N. P. Proukakis, Time-of-flight expansion of binary Bose-Einstein condensates at finite temperature, New J. Phys. 20, 053004 (2018).

[34] S.-W. Su, S.-C. Gou, I.-K. Liu, I. B. Spielman, L. Santos, A. Acus, A. Mekys, J. Ruseckas, and G. Juzeliūnas, Positiondependent spin-orbit coupling for ultracold atoms, New J. Phys. 17, 033045 (2015).

[35] V. E. Lembessis, Artificial gauge potentials for neutral atoms: An application in evanescent light fields, J. Opt. Soc. Am. B 31, 1322 (2014).

[36] M. Mochol and K. Sacha, Artificial magnetic field induced by an evanescent wave, Sci. Rep. 5, 7672 (2015).

[37] R. Sachdeva and Th. Busch, Creating superfluid vortex rings in artificial magnetic fields, Phys. Rev. A 95, 033615 (2017).

[38] V. E. Lembessis, A. Alqarni, S. Alshamari, A. Siddig, and O. M. Aldossary, Artificial gauge magnetic and electric fields for free two-level atoms interacting with optical ferris wheel light fields, J. Opt. Soc. Am. B 34, 1122 (2017).

[39] J. R. Abo-Shaeer, C. Raman, J. M. Vogels, and W. Ketterle, Observation of vortex lattices in Bose-Einstein condensates, Science 292, 476 (2001).

[40] J. W. Reijnders and R. A. Duine, Pinning of Vortices in a BoseEinstein Condensate by an Optical Lattice, Phys. Rev. Lett. 93, 060401 (2004).

[41] S. McEndoo and Th. Busch, Small numbers of vortices in anisotropic traps, Phys. Rev. A 79, 053616 (2009).

[42] N. Lo Gullo, Th. Busch, and M. Paternostro, Structural change of vortex patterns in anisotropic Bose-Einstein condensates, Phys. Rev. A 83, 053612 (2011).

[43] J. Stockhofe, S. Middelkamp, P. G. Kevrekidis, and P. Schmelcher, Impact of anisotropy on vortex clusters and their dynamics, EPL (Europhysics Letters) 93, 20008 (2011). 
[44] M. Tsubota, K. Kasamatsu, and M. Ueda, Vortex lattice formation in a rotating Bose-Einstein condensate, Phys. Rev. A 65, 023603 (2002).

[45] K. Kasamatsu, M. Tsubota, and M. Ueda, Nonlinear dynamics of vortex lattice formation in a rotating Bose-Einstein condensate, Phys. Rev. A 67, 033610 (2003).

[46] L. Wen, H. Xiong, and B. Wu, Hidden vortices in a BoseEinstein condensate in a rotating double-well potential, Phys. Rev. A 82, 053627 (2010).

[47] L. Wen, Y. Qiao, Y. Xu, and L. Mao, Structure of twocomponent bose-einstein condensates with respective vortexantivortex superposition states, Phys. Rev. A 87, 033604 (2013).

[48] C. J. Pethick and H. Smith, Bose-Einstein Condensation in Dilute Gases (Cambridge University Press, 2008).

[49] F. Dalfovo, S. Giorgini, L. P. Pitaevskii, and S. Stringari, Theory of Bose-Einstein condensation in trapped gases, Rev. Mod. Phys. 71, 463 (1999).

[50] M. Cheneau, S. P. Rath, T. Yefsah, K. J. Günter, G. Juzeliunas, and J. Dalibard, Geometric potentials in quan- tum optics: A semi-classical interpretation, EPL 83, 60001 (2008).

[51] M. Feit, J. Fleck Jr., and A. Steiger, Solution of the Schrödinger equation by a spectral method, J. Comput. Phys. 47, 412 (1982).

[52] J. Polo, V. Ahufinger, P. Mason, S. Sridhar, T. P. Billam, and S. A. Gardiner, Analysis beyond the thomas-fermi approximation of the density profiles of a miscible twocomponent bose-einstein condensate, Phys. Rev. A 91, 053626 (2015).

[53] The numerical data points are found by adjusting a Gaussian distribution onto the derivative of the density profile, which give us a mean value and variance for each fitting point, $\mu \pm \sigma$. We specifically calculate $\partial_{z} \phi_{1}(z) \approx \mathcal{N}(\mu, \sigma)$ for each $z_{0}$, with $\phi_{1}(z)=\int d x|\psi(x, z)|^{2}$ and $\mathcal{N}(\mu, \sigma)$ being the normal distribution.

[54] J. Schloss, P. Barnett, R. Sachdeva, and Th. Busch, Controlled creation of three-dimensional vortex structures in Bose-Einstein condensates using artificial magnetic fields, Phys. Rev. A 102, 043325 (2020). 